\title{
Hardware and Software Requirements for a Picture Archiving and Communication System's Diagnostic Workstations
}

\author{
D.R. Haynor, D.V. Smith, H.W. Park, and Y. Kim
}

\begin{abstract}
Electronic systems (picture archiving and communications systems [PACS]) for image and multimedia data distribution, archiving, and transmission, represent the future of radiology. The workstation is the point of contact between a PACS and the radiologist or referring physician. Therefore, the acceptance of PACS is highly dependent on workstation functionality and performance. This paper, based on our experience in evaluating commercial workstations and on a review of recent literature, describes hardware and software requirements for diagnostic workstations that could be used for making primary diagnoses in a radiology department. Requirements for PACS workstations for use in referring clinics are also briefly described. These workstations must be able to handle the large volume of images to be viewed efficiently, add new functionality to improve the productivity of physicians, technologists, and other health care providers, and provide enough flexibility to allow the electronic systems to grow as medical imaging technology evolves.

Copyright $\odot 1992$ by W.B. Saunders Company
\end{abstract}

KEY WORDS: PACS, diagnostic workstation, workstation user interface, image display, image processing, image storage, image transmission, clinical workstation.

$\mathbf{I}^{\mathrm{N}}$ N RECENT YEARS, medical procedures have become more complex, while financial pressures for shortened hospital stays and increased efficiency in patient care have increased. As a result, several shortcomings of present film-based systems for managing medical images have become apparent. Maintaining film libraries is labor intensive and consumes valuable space. Film is expensive (typical costs for a 350-bed hospital are on the order of $\$ 700,000 /$ year). Because only single copies of radiological examinations exist, they are prone to being lost or misplaced, thereby consuming additional valuable time and expense. It is difficult for radiologists to deliver diagnoses in a timely fashion.

An electronic system for image archiving, transmission, and viewing (picture archiving and communications system [PACS]) offers a solution to these problems. Figure 1 is a conceptual diagram of a PACS. Multiple copies of images can exist and can be viewed simultaneously without conflicts. Image loss can be eliminated, and film costs can be greatly re- duced. The space required for archiving can be significantly reduced. In addition, many enhancements become possible. Image processing or artificial intelligence can be used to improve the conspicuity of lesions or to screen images for specific abnormalities. The task of comparing multiple radiological studies on the same patient can be made significantly easier. Interactive on-line reference databases, containing text and images, can be developed.

At present, no fully or largely digital radiology departments exist, although several are in the planning stage. ${ }^{1,2}$ The problem of achieving adequate network and display speed, display resolution, and archival capacity at an acceptable cost has proven to be more difficult than was originally anticipated.

It is likely, however, that new developments in hardware will allow significant reductions in the costs of transmitting, archiving, and viewing medical images over this decade, to the point where electronic systems will become costcompetitive with conventional systems. If, at the same time, software can be developed that significantly enhances the productivity and diagnostic accuracy of individuals viewing images electronically rather than on conventional film, the attractiveness of PACS will be further enhanced, and hospitals and radiology departments are likely to invest in the new technology.

This paper discusses the requirements for a radiology workstation suitable for making primary diagnoses in the radiology department. The important characteristics from a technical standpoint are good image quality, a friendly, reliable and intelligent user interface, sufficiently rapid response time, and productivity aids that enable the user to complete his or her diagnostic task successfully. The set of require-

From the Center for Imaging Systems Optimization, University of Washington, Seattle, WA; and the Department of Radiology, Madigan Amy Medical Center, Tacoma, WA.

Address reprint requests to Yongmin Kim, PhD, Department of Electrical Engineering, FT-10, University of Washington, Seattle, WA 98195.

Copyright $@ 1992$ by W.B. Saunders Company

0897-1889/92/0502-0007\$03.00/0 


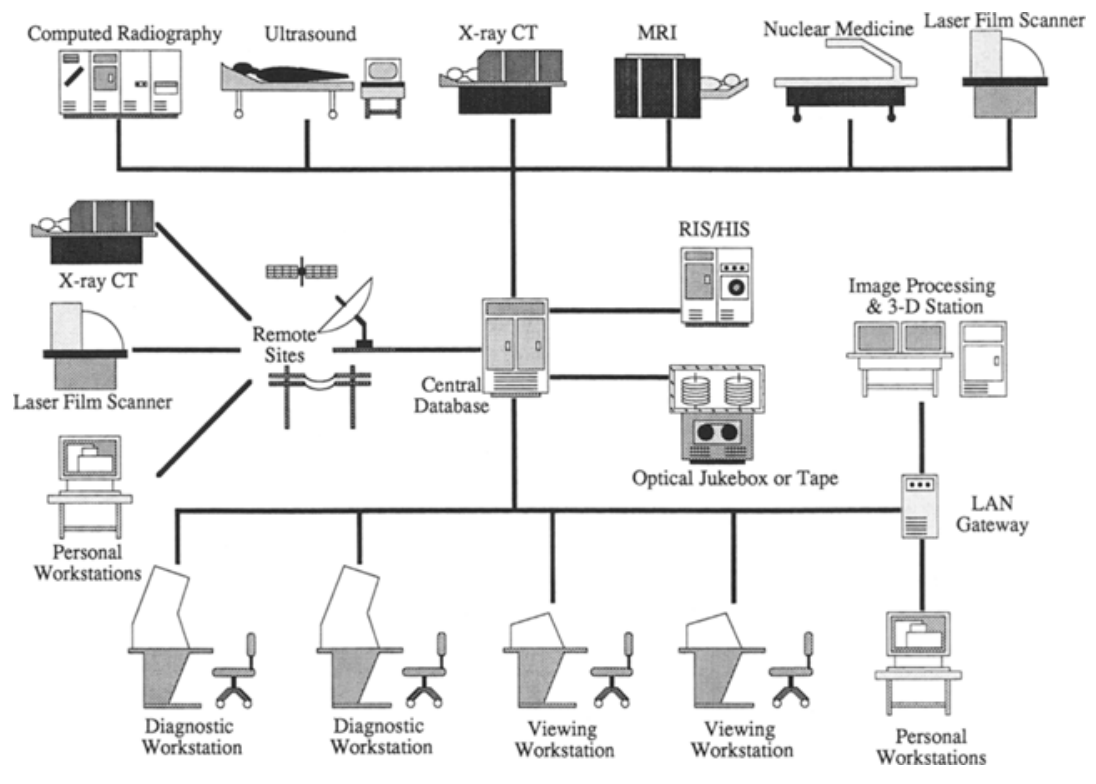

Fig 1. A schematic diagram showing major PACS components and connections. ments described below, which go beyond any currently commercially available systems, are drawn from our own work ${ }^{3,4}$ and from a review of published literature. For other recent reviews of workstation requirements, the reader is referred to references 5 to 7 . Current developments in workstation design for PACS and medical imaging are described in the annual proceedings of the Society of Photo-optical Instrumentation Engineers (SPIE)-sponsored conferences on medical imaging. ${ }^{8}$

\section{THE DIAGNOSTIC VIEWING TASK IN A CONVENTIONAL RADIOLOGY DEPARTMENT}

A description of the diagnostic viewing/ reporting session, as it occurs in a conventional department, is a useful starting point for an analysis of workstation requirements, ${ }^{9-11}$ because the radiologist working at a PACS workstation will have to perform equivalent tasks. The diagnostic process is the most complex interaction any individual has with the image viewing/distribution system and so its requirements are the most stringent. The needs of other individuals using the PACS, or of the radiologist using it for other purposes, are generally subsets of the functionalities that must be supplied to make diagnostic sessions productive and pleasant (except for certain specialized purposes such as radiation therapy planning). Any steps or delays that significantly slow down the reporting process will also be a problem in busy clinical environments.
Although the radiologist is frequently involved in the conduct or supervision of a radiographic examination, he usually views the images only when they have been acquired and arranged into a format suitable for viewing and reporting. In some cases (eg, military hospitals), however, he may also be responsible for arranging the films on the viewing device (light box or alternator). In a diagnostic session, he views a sequence of examinations and dictates a report for each. He usually follows a predetermined order for the examinations, operating from a worklist of examinations that have been routed to him. This worklist is generated by ancillary personnel (possibly the people who arrange the films initially) and typically contains all unreported studies for a particular modality or body region, arranged roughly in chronological order of acquisition. In many cases, these studies are arranged according to a specific layout protocol. For example, in the case of a two-view chest study with comparison study, the images might always be put up in the order (left to right): old frontal view, new frontal view, new lateral view, old lateral view. Plain films are generally put up in a specific orientation (eg, patient's right to the viewer's left). In cases in which the radiologist arranges the films himself, he may follow his own set of layout rules.

The alternator is a mechanical device for temporary storage of large numbers of images for viewing. It contains 20 to 60 panels, each of which can hold up to four $14 \times 17$-in films $(1$ 
film $=1$ chest image, 12 to 15 computed tomography or magnetic resonance [CT/MR] images, or 15 to 30 ultrasound images). Any two consecutive panels can be viewed simultaneously, so that up to eight $14 \times 17$-in films can be viewed at once. The radiologist can advance to any one of the other viewing panels by pressing a button. The panels currently being viewed are then replaced in a large storage bin and the requested panels are selected and moved up to viewing position, all automatically. The longest period of time required to move from one pair of panels to a second pair is about 10 seconds for modern "random access" alternators, and is about 6 seconds for adjacent panels. An eightscreen viewbox, which is equivalent to two alternator panels, is an alternative viewing device that may be used if the radiologist must arrange his own films.

Near each alternator is a bin containing the film jackets for each patient who has a study on that alternator. These jackets contain all of the old films for that patient, most of which are unrelated to the current study. Usually one or more of the most recent prior studies of the same type as the current study is mounted with that study by the file room staff. ${ }^{12-14}$ For instance, if the patient's current examination is an examination of the chest, the most recent previous chest examination, as well as up to two older studies, are also mounted on the same or adjacent panel on the alternator.

Many cross-sectional studies (CT/MR) require two, and often three or four panels, because of the large number of images. Such studies must frequently be compared with old studies of similar size. The film-based system is particularly awkward with studies containing multiple series of images (and even more so when comparison to a second study must be made). Here the radiologist must compare each image with other images, obtained with different acquisition parameters, which represent the same anatomic location or "slice" in the patient, or with images at adjacent locations acquired with the same parameters.

The radiologist will generally view all the images in the examination at least once in a sequential fashion. After proceeding through the entire study, he may wish to go back to a particular subset of images that, for example, contain significant pathology. ${ }^{15} \mathrm{He}$ may mark these images of findings (with a pencil or wax crayon) for reference during reporting and for later viewing by referring physicians. An effective computer interface thus must allow image marking and rapid viewing in a sequential mode, as well as convenient random access to selected images. Availability of a low-resolution overview of the entire study is also useful. Proceeding from image to image (either sequentially or in a random order) has been termed navigation. ${ }^{15}$

Occasionally the radiologist will want or need to consult journal articles, textbooks, etc, for assistance in the interpretation of a particular finding. The frequency with which he does this is directly related to the ease of access to such materials.

In the film-based system, the extraction of quantitative information from images is limited and relatively simple. Distances may be estimated by direct measurement (plain film) or by comparing them with a scale incorporated into the image (ultrasound, CT, MR). Measurement of the areas or volumes of irregular surfaces, although desirable, is too tedious to be practical on a routine basis. For CT, the dynamic range of the image exceeds that of film, and so a given study is frequently photographed with two or three window/level settings (ie, different image intensity-versus-film-density look-up tables).

The radiologist must dictate a diagnostic report for each examination. Some overhead is associated with the process of identifying the examination to the dictation system. This may include identifying the patient, the examination, the date and time of examination, and the personnel involved in the execution and interpretation of the examination. Dictation is generally done simultaneously with viewing. The report is then transcribed by a transcriptionist. Transcription typically takes 24 to 48 hours; in urgent cases, the referring physician is contacted directly with the findings. Copies of the final report, when signed by the radiologist, are sent to the patient's chart, to the referring physician, to the radiology file room, and to the billing office. The fileroom stores the report with the associated films for future reference. When a radiology information system (RIS) is used, an electronic copy of the report may be available at an RIS workstation as soon as transcription is complete. 


\section{DISPLAY AND STORAGE REOUIREMENTS FOR DIAGNOSTIC WORKSTATIONS}

The previous section described the tasks that radiologists and fileroom clerks perform in conventional diagnostic film reporting. The ability to support these tasks, or their equivalent, is a minimum requirement for PACS. Although the most significant economic benefits to the hospital from PACS may actually accrue outside the radiology department, ${ }^{16,17}$ no system that is unacceptable to radiologists is likely to be fully successful. This implies that diagnostic accuracy and radiologist productivity must be at least equivalent to that of conventional systems with PACS if the large investments required are to be made. This has important consequences for workstation design.

\section{Image Formats}

The usual sizes for MRI/ultrasound or CT images are $256 \times 256$ or $512 \times 512$, respectively. Nuclear medicine images range from $64 \times 64$ to $256 \times 256$. Magnetic resonance imaging and CT images are 12 bits deep; ultrasound and nuclear medicine images are typically 8 bits deep. Computed radiographs and laser-digitized radiographs are much larger, approximately $2,000 \times$ 2,000 , and 10 to 12 bits deep. Conversely, individual computed radiographic studies contain 2 to 4 images, while CT, MR, and ultrasound studies may contain from 10 to over 100 images. The result is that individual studies contain between 5 and 20 Mbyte of information. ${ }^{18}$

Accompanying alphanumeric information giving information about the patient, the study, and each image is complex but amounts to only a few kilobytes per study. A format for the complete data set consisting of the demographic information, the modality information, and the images, is defined by the American College of Radiology and the National Electrical Manufacturers Association (ACR/NEMA) message or data set structure standard. ${ }^{19}$ The ACR/NEMA standard also specifies a physical interface protocol for connecting image acquisition devices to a network via a network interface unit. This connection standard must be supported by any PACS vendor who wishes to have full digital interfaces to multiple acquisition devices, some of which may be from other vendors.

\section{Display Characteristics}

The viewing of computed radiographic (plain films) and cross-sectional studies imposes rather different demands on a workstation. The diagnostic workstation, however, should be usable for either type of study, and even for viewing both types simultaneously.

Viewing of plain radiographs places the greatest requirements on the spatial resolution of the screen, which is only partially described by giving the number of pixels in the display. ${ }^{20}$ For the purposes of this discussion, a medium resolution display is approximately " $1 \mathrm{~K}$ " or $1,000 \times$ 1,000 pixels (eg, a typical scientific/engineering workstation display), while a "high resolution" display is approximately " $2 \mathrm{~K}$ " or $2,000 \times 2,000$. A number of studies have been performed to assess spatial resolution requirements for accurate plain film diagnosis, focusing primarily on requirements for chest and musculoskeletal radiology. At least two recent large, carefully performed receiver-operating characteristic (ROC) studies have shown statistically significant differences in the detectability of certain types of lung lesions (pneumothoraces and interstitial lung disease) on digitized x-ray images, displayed on high resolution monitors, ${ }^{21,22}$ when compared with detectability of the lesions on the original images. Detectability of other lesion types (lung nodules, mediastinal masses, apical scarring, etc) was equivalent. Two other studies $^{23,24}$ have shown that the visualization on digitized conventional radiographs of nondisplaced fractures of the extremities and of metaphyseal fractures in children was impaired if the pixel size exceeded $0.16 \mathrm{~mm}$ (equivalent to $2 \mathrm{~K}$ resolution). Even more stringent resolution requirements were reported for the reliable detection of subperiosteal bone resorption, 25 although the clinical importance of assessing mild degrees of bone resorption is uncertain. The paper by Slasky et $\mathrm{al}^{22}$ also suggests that the performance of observers viewing digitized radiographs that were then printed on film was slightly superior to their performance on an interactive high-resolution display with the same nominal resolution. Collectively, these studies suggest that maintaining the same diagnostic accuracy for subtle plain film findings will require the use of at least some $2 \mathrm{~K}$ monitors as a component of a diagnostic workstation, and that 
the effective spatial resolution of the current generation of $2 \mathrm{~K}$ monitors may be somewhat less than the nominal value. These papers do not directly support the requirement that all the monitors be of $2 \mathrm{~K}$ resolution; probably only a subset (those used for viewing the current image, and perhaps one additional monitor for comparison) need be high resolution, as long as images are archived at high resolution and real-time zoom is supported on the medium resolution monitors. Other studies have suggested that high-resolution displays are adequate for studies of the genitourinary and gastrointestinal systems. ${ }^{26,27}$

The screen size should be at least 19 inches to provide adequate space for viewing multiple images; because of the varying levels of background illumination in viewing rooms, a luminance of at least 60 foot-Lamberts is desirable (conventional light boxes have a luminance of 200 to 400 foot-Lamberts). ${ }^{28}$ The ability to measure, and monitor on a continuing basis, the physical characteristics of the workstation displays is highly desirable. While some measurements are possible only with expensive equipment, Roehrig et $\mathrm{al}^{29}$ have described a fairly complete set of performance tests that are reasonably simple to perform with a photometer. Workstation software should be available to display the images needed for these or similar quality assurance tests. Appropriate standards for display performance, particularly for the newer high-resolution displays, do not yet exist. The dynamic range (1000:1 or more) of many medical images requires 10 to 12 bits of contrast resolution, and diagnosis requires adjustment of the window and level (contrast and brightness) values of the display at interactive speeds. As a practical matter, therefore, a 12-bit-deep frame buffer is required. The availability of one or two extra bit planes for graphics overlays (annotations, regions of interest, etc) is desirable but not absolutely necessary. Displays must be a minimum of $60 \mathrm{~Hz}$ (noninterlaced) to achieve minimal flicker. Because peripheral vision is more sensitive to flicker than central vision, monitor quality with multiple-screen workstations becomes even more important than with single-screen devices, and refresh rate requirements are slightly higher $(>70 \mathrm{~Hz}) .{ }^{28}$ When multiple monitors, possibly with different dis- play resolutions, are provided, brightness, phosphor color, phosphor decay time, and other characteristics of the individual monitors should be as similar as possible. ${ }^{6}$

The number of monitors required for a diagnostic workstation remains uncertain, with current implementations including between 4 and 8 monitors (the latter thus being comparable with a standard alternator in terms of total viewing area). A smaller number of monitors, while lowering the cost of the workstation, places an additional burden on the workstation designer, because the tasks of study comparison and movement within studies become much more difficult, particularly for large cross-sectional examinations. By making it possible for the radiologist to view a large number of images simultaneously, simply by moving his head or eyes, a workstation with a large display area reinforces the radiologist's mental model of the spatial layout of the images in the study, and reduces the number of workstation operations required to view the entire study, as well as the cognitive demands of remembering the contents of multiple off-screen images. Conversely, if a smaller number of images are available for simultaneous viewing, navigation within the study must be both intuitive and extremely rapid. Workstations designed exclusively for viewing of computed radiographs need to display fewer images at a given time, and four monitors are likely to be sufficient in most cases.

When a study is first accessed, 2 seconds or less is an adequate time for each complete screen to fill. Once a study has been identified as the current study, and loaded into the local high-speed semiconductor memory, paging back and forth within the study should be nearinstantaneous ( $<1$ second per screen). ${ }^{12,30}$

\section{Memory and Disk Requirements}

Requirements for workstation memory and disk size are influenced by study size and network design. Because a typical study ranges from 5-20 Mbytes in size, and because one might frequently wish to correlate up to three studies simultaneously, high-speed access to 40 to $60 \mathrm{Mbytes}$ of data is required. In some cases (eg, frequent viewing of three-dimensional or large digital angiography studies), up to 100 Mbyte might be required for selected worksta- 
tions. If a very high-speed network (signaling rate $>300 \mathrm{Mbit} / \mathrm{s}$ ) is used, there is no need to store studies other than those currently being viewed locally, provided that a sufficiently fast central file server is available. Recent studies have suggested that this design may actually be the most cost-effective PACS design within a few years. ${ }^{18}$ If the network is slow, on the other hand, it is desirable to prefetch studies on the worklist (and the appropriate comparison studies) to the local disk, and local Winchester requirements grow accordingly to the 500- to 1,000-Mbyte range. In any case, the speed of image transfer from disk to memory (whether from a local or central disk) must be such that high-resolution screens ( $\sim 6$ to 8 Mbyte of data per screen) can fill in approximately 2 seconds. Currently, these speeds are achievable only by parallel transfer disks. The burst speed required for a central file server in a medium-sized hospital (300 beds, performing 100,000 studies per year) can be estimated to be about 20 Mbyte/s, as the possibility of multiple simultaneous requests must be considered.

\section{Ergonomics}

While many of the ergonomic issues associated with single-monitor workstations are well understood from experience with personal computers and conventional workstations, the introduction of multiple monitors creates some new problems. Bezels between multiple monitors should be as small and nonintrusive as possible, as they reduce the visibility of portions of the screens. Most designs for multiple-monitor workstations envisage two rows of monitors; the top row should be as low as is feasible to minimize the amount of neck extension necessary to view them. If a mouse or other interactive device is used for menu selection, moving images, etc, the device should be optimized for working on large display surfaces. The cursor should move easily within and between monitors in a smooth continuous manner and should always be visible during its movement. The workstation must be physically robust, resistant to spills and collisions, and look attractive. Because multiple workstations will typically be in use in a single room, noise (fans) should be minimized. It is acceptable and even desirable to divide the workstation into two components-a computer/ storage system and a display device-as long as the allowable distance between them is adequate (a minimum of $200 \mathrm{ft}$ ). Additional considerations relating to room layout, particularly those relating to lighting and the placement of multiple workstations, are discussed by Horii et al. ${ }^{31}$

\section{IMAGE PROCESSING}

Certain image processing functions are necessary for diagnostic workstations, while the use of others remains unproven. We begin with a discussion of the low-level functions that most designers of imaging workstations and consoles for CT and MR scanners have found useful.

Window and level adjustment: The user must be able to adjust the window and level (lookup table) governing the display of a single image, all images on a single monitor, or an arbitrarily selected subset of the displayed images. Inverting the gray scale for a selected set of images is occasionally useful. Appropriate default window/level settings (user adjustable) should be provided for each image, reducing the amount of time the user must spend on window/level adjustment. These settings could be imagespecific and generated by a technologist at the time of image acquisition, much like the conventional film-based system, modality- and bodyspecific using user-configurable rules (like current computed radiography systems), or could be generated automatically using more complex methods. ${ }^{32}$

Zoom and roam: The workstation must be able to enlarge the image two or four times and display it by simple replication of pixel values. The ability to enlarge the image two or four times and display it by interpolating intermediate pixel values in a smooth continuous manner is also useful. Providing intermediate levels of zoom, although useful, is not essential. If the entire image is not viewable at the specified screen resolution, it must be possible to smoothly roam the entire image.

Image reorientation: It is sometimes desirable to be able to change image orientation. This usually is done because of some error made during image acquisition and typically the ability to flip the image or rotate it by some multiple of $90^{\circ}$ is all that is required. It should be possible to save the new orientation. 
Digital magnifying glass: It must be possible to roam a user-specified rectangle over the image and provide two- or fourfold magnification of the central portion of the image within the rectangle.

Image mensuration: The user must be able to compute point-to-point distance measurements with automatically calibrated, user-selectable scales. He also must be able to perform angular measurements and calculate the area and perimeter measurement of elliptical and rectangular regions of interest. It should be possible to compute statistics (histogram, mean intensity, standard deviation, range, number of pixels) for any selected region of interest. It should be possible to designate irregular regions of interest with a cursor and perform similar measurements. The workstation should compute and display these functions for multiple measurements simultaneously on the same image and save them (at the user's option) as a part of a graphics overlay that can be toggled on and off. If an overlay is saved, viewers of the annotated images should be notified of the existence of the overlay.

Three-dimensional viewing: Two-dimensional images taken as parallel slices through a threedimensional object can be reformated and viewed in a variety of ways to better display three-dimensional relationships. This capability is useful for some diagnostic problems and for surgical and radiation therapy planning. At a minimum, it is desirable to have off-line workstations that can create sets of views of threedimensional objects from stacks of slices through them; if these workstations have the appropriate network connections, they can be viewed on other workstations simply as another type of image set. Alternatively, some or all workstations may be provided with the ability to do three-dimensional reformatting and viewing directly.

Spatial registration of studies: Comparison of different studies of the same body part (same modality at different times or different modalities) can be greatly facilitated if corresponding anatomical points can be identified. For crosssectional studies, at least, this is primarily a matter of relating two three-dimensional Cartesian coordinate systems (one for each study) to each other. At least two general approaches are possible. In one, a user is responsible for identifying sets of corresponding three-dimensional points. Another approach uses computer methods to identify corresponding points. Once a set of corresponding points has been identified, the appropriate coordinate transformations can be calculated and any point on the first study can be matched with a unique point on the second study; as one views the new study, the equivalent slice (or nearest approximation) would come up on the old study, making the detection of change easier.

Higher-level image processing functions: Providing the radiologist with a large set of complex but low-level image processing functions (mathematical morphology, filtering, active contour methods, image segmentation algorithms, etc) is not likely to be useful; radiologists are unfamiliar with these techniques and in any case do not wish to spend large amounts of time optimizing them for a specific radiological problem. Some specific tools, such as unsharp masking, may sometimes be useful in "salvaging" computed radiographs. ${ }^{33}$ However, software packages can be created by workstation developers to aid the radiologist with certain specific diagnostic tasks. Examples of this would include modalityspecific analysis packages (nuclear medicine, cardiac MRI, MR angiography, etc) and methods to screen for certain specific abnormalities (microcalcifications in mammograms, pulmonary nodules, etc). These screening methods are typically run without user intervention and point the radiologist to possible abnormalities in the image. If the false-positive rate of a screening method is acceptable, the rate of radiologist "misses" can be greatly reduced. This type of image processing in computer aided diagnosis was recently reviewed by MacMahon et al. ${ }^{34}$

\section{THE WORKSTATION USER INTERFACE}

Raw display and image processing speed, although important, is insufficient. If selecting a particular workstation function is awkward or time-consuming, the fact that it can be performed rapidly, once selected, is irrelevant. The nature of radiological practice places a high premium on the ability of the radiologist to rapidly perform a limited set of complex tasks. This principle suggests that reasonable default 
choices must be made by the system whenever possible. Some of these may be site-specific and configurable by the system operator, while others may be customized for the individual user. Tasks that can be anticipated (eg, fetching the next study on a worklist) should be performed by the system automatically in the background. Status messages should be provided for processes that are expected to take a long time. As workstations become more complex, the availability of on-line context-specific help and of system tutorials become important.

General: It is assumed that some type of direct manipulation interface will be provided. This seems appropriate in view of the fact that an obvious metaphor (namely, the conventional film-based system) already exists for the image viewing process. Shortcuts, possibly keyboardbased, must also be provided for the experienced user, and an "undo last command" function should be provided. Because the radiologist must frequently interrupt his work, it should be possible to save the current working environment to allow access to a new patient's images. It should be possible to save and then restore multiple working environments.

We now review some specific requirements in an order roughly corresponding to the tasks described above that are performed during a conventional film-reading session.

Logon and logoff: The user should be required to $\log$ on to the workstation, using a password, at the start of a viewing session. This improves system security and allows display options, access and modification privileges, layout options, etc, to be user- or site-specific. Logoff after a prolonged period of inactivity should be automatic, but the current working environment(s) should be saved for each user.

Study selection: The user interface must support a clinically useful view of the image database. It must be possible to select studies by patient number or name or portions thereof. Once a patient has been selected, differing views of that patient's folder should be available, with studies sorted by modality, date and/or body part (at the user's option). An innovative "anthropomorphic" view of patient's folders is described by Roger et al. ${ }^{11}$ When the study of interest has been identified, possible comparison studies should be automatically identified and ranked in order of interest according to site- and modality-specific rules. If the radiologist is using a worklist, it should be possible to automatically advance to the next study on the worklist, or to view the entire worklist at one time.

Study layout: Once a study is selected, a default study layout (the way in which the study images are arranged on the monitors) should be invoked. The goal should be to emulate the process performed by the file clerk who places films on the alternator. This would include the selection of appropriate monitors for each image in the current study and comparison studies. These protocols are dependent on modality and body part and should be, within reasonable limits, user-configurable. Default window/level settings, image orientation, and image magnification should also be specified by modifiable protocols.

Image rearrangement: Providing useful ways to view multi-image studies is probably the single most challenging aspect of the user interface, and is an area of considerable research interest at the present time. ${ }^{15}$ It is useful to visualize the total set of images currently being viewed as divided into studies (typically, a "current" study and other comparison studies), with each study divided into acquisition series and each series made up of a linearly ordered set of images. Methods must be provided for selecting subsets of images at each of these levels (study/series/ image) and performing particular operations (rearrangement, image processing, etc) on the selected subset. A minified overview (gallery or survey mode) of the entire image set, from which image selection and arrangement can be performed, is useful, but it must be possible to toggle back and forth between the overview image and the full-resolution images nearly instantaneously if this viewing mode is to be useful. Because radiologists view studies both in sequential and "random access" fashion, both of these modes of image navigation must be facilitated. The ability to mark images of interest is useful both to facilitate moving around in large studies and to identify images for examination by referring physicians and other radiologists. Similarly, the ability to perform spatial 
registration of different series or studies can simplify the comparison task greatly. Display speed, network speed, and size of workstation memory interact strongly here with the software of the user interface.

Cine viewing: The ability to arrange images in stacks and then view them as a movie loop (at selectable speeds) is quite useful, particularly if the process of assembling the stack is easy.

Image annotation and marking: The system stores a great deal of information about each study and image; the user must be able to toggle the visibility of this information on and off. The placing of marks on films is an important way in which physicians communicate with one another about images. These marks may be used to identify significant images or to point to or encircle significant areas of pathology within an image. The workstation must offer the radiologist or other physician the capabilities to mark images, create pointers and notes, and store the annotations as an overlay. Users viewing those images at a later time should be notified that annotations are available.

Consultation: The radiologist spends a significant proportion of his working day consulting with referring physicians. The PACS workstation should provide tools to facilitate this task. These include the ability to set the current working environment aside and examine a different study and/or patient. If the radiologist is unfamiliar with a particular case, he may wish to refer to images that have been marked as being of particular interest. He must have quick access to all types of information about the consultation case, including the spoken or typed radiology report for the examination. If consultation is performed for a large group, the ability to direct an image to a large-screen display device may be useful. If consultation is performed with a referring physician over the telephone, the ability to draw an arrow or manipulate a cursor on the other workstation is useful.

Hard copy generation and creation of teaching files: The radiologist will occasionally want to print the contents of a selected monitor on a hard copy device. The ability to print textual information (worklist, old reports, etc) is also useful, as is the ability to produce 35 -mm slides for teaching or archival purposes. The ability to mark studies and selected images for reference purposes (individualized teaching files) will be one of the most attractive aspects of PACS as the size of on-line image databases grow, because they offer the potential for making the radiologist "smarter" by increasing the amount of accessible knowledge he has at his fingertips. Several interesting projects attempting to link reference databases to PACS have already been initiated. ${ }^{35-37}$

Use of color: Color displays are routinely used in nuclear medicine, and color photographs are used for Doppler imaging of flow with ultrasound. Selected workstations, therefore, should have color capabilities, with one or two color monitors and additional grayscale monitors. Some degradation in spatial resolution and luminance characteristics of the color monitors is to be expected.

RIS integration: Historically, PACS have been developed independently of Radiology Information Systems (RIS) and Hospital Information Systems (HIS), text-based systems that have already been implemented in essentially all American hospitals. In the future, integration between PACS, the HIS, and the RIS will have to be much tighter for several reasons. Considerations of real estate and expense suggest that PACS terminals should be able to double as RIS and HIS terminals. Access to previous reports is vital for image interpretation by both radiologists and referring physicians, and these reports are available only through the RIS. The RIS and HIS contain vital ancillary information about patients and their medical problems that can increase the accuracy of diagnosis and is needed for automatic worklist generation. Because the trend of independent development of PACS and RIS/HIS may very well continue, strict adherence to the emerging ACR/NEMA guidelines on RIS/PACS interfaces will be required.

Voice recognition: The use of a voice-driven interface to a PACS workstation is still unproven. However, the availability of voice-based report transcription, which could eliminate the long delays between diagnosis and transmission of a report to a referring physician, will have an enormous impact on the practice of radiology. Voice recognition hardware capable of dealing 
with continuous speech at reasonable prices is expected to become available during this decade.

Teaching files and reference cases: Efforts are already underway at multiple institutions to create a variety of $\mathrm{CD}$ - or videodisc-based teaching resources in radiology. These resources typically include a mixture of radiological images, anatomical photographs, photomicrographs, text, line drawings, etc. In the future, one could imagine large amounts of radiological knowledge (in both textual and image form) being available on-line for immediate consultation. If a user could interact with such a resource directly from his workstation, he might well be able to offer more specific or useful diagnoses. No standards exist in this area as yet, and it is difficult to predict the final forms these resources will take. Thought should be given to providing "hooks" within workstation software to make access to these interactive knowledge bases possible.

\section{CLINICAL WORKSTATION}

The needs of the user (usually a referring physician) viewing images outside the radiology department are broadly similar to those of the radiologist reporting a study. Ideally, the referring physician would like to have access to the radiologist's report at the time he views a study. He may wish to view primarily those images the radiologist has marked as containing interesting information. He will typically have access to workstations with fewer monitors than those in the radiology department and he will be less familiar with the workstation user interface. If he examines images before they are seen by a radiologist, he may wish to record his impression so that, if there is a significant variance, the radiologist can contact him for further discussion of the case.

The diversity of clinical environments translates into a broad diversity of workstations, ranging from units comparable with diagnostic radiology units for certain specialty clinics to PC-like workstations augmented with additional displays, memory, and image processing hardware. The total cost of the workstations situated outside of the radiology department will typically exceed those within the radiology department, ${ }^{18}$ although the unit costs are less. Much of the same software functionality should be provided so that the referring physician, who will also use workstations in the radiology department, has only a single interface to learn. Because referring physicians will be less frequent users of PACS than radiologists, the importance of a consistent and friendly user interface and the availability of on-line help are magnified.

The needs of particular clinical environments must be analyzed on a case-by-case basis. For nursing stations, for example, a pair of 1,000 monitors will usually be adequate. The use of a keyboard may not be convenient or even desirable if a relatively limited set of review functions are needed. Alternate input devices may be more suitable in such cases. There will be an environment-specific view of the database (for example, the default display would be of the patients currently staying on a particular floor or scheduled to be seen in a particular clinic). This view is typically configured by the system operator, and requires good integration with the HIS in order to be effective (because only the HIS contains information about patient beds or clinic appointments).

\section{CONCLUSIONS}

PACS represent the future of radiology in the modern hospital. The declining costs of PACS components, the increasing sophistication of software, and the better integration of PACS components will make hospital-wide systems a reality within this decade.

At the same time, the potential of PACS has barely been tapped. Workstation and database software can be developed that can substantially increase physician productivity, improve diagnostic accuracy, and make a large amount of knowledge and patient information available on-line to the physician in the hospital or in his office. Development of these features will require a substantial period of prototype development and testing in close collaboration with end-users. It is expected that hardware improvements and cost savings will be incorporated in parallel during this development period. This paper has described some of the directions in which this development must proceed. 


\section{REFERENCES}

1. Huang HK, Kangarloo H, Cho PS, et al: Planning a totally digital radiology department. Am J Roentgenol $154: 635-639,1990$

2. Goeringer F: Medical diagnostic imaging support systems for military medicine. SPIE Medical Imaging V 1444: 340-350, 1991

3. Haynor DR, Weghorst S, Saarinen AO, et al: Clinical evaluation of PACS workstations-methodology and results. SPIE Medical Imaging IV 1234:408-417, 1990

4. Haynor DR, Kim Y: Digital radiology system requirements document. Center for imaging system optimization, University of Washington, Seattle, WA, 1991

5. MDIS: Performance Work Statement of the Medical Diagnostic Imaging Support System. US Army Eng Div, Huntsville, AL, No. DACA87-90-R-0058, 1990

6. Arenson RL, Chakraborty DP, Seshadri SB, et al: The digital imaging workstation. Radiology 176:303-315, 1990

7. Pizer SM, Beard DV: Medical image work stations: functions and implementation. J Digital Imaging 2:189-93, 1989

8. Proceedings of the SPIE Conferences on Medical Imaging: Society of Photo-optical Instrumentation Engineers, Bellingham, WA, 1982-1992

9. Levin K, Horii S, Mun SK, et al: Analysis of data assembling activities for radiologists and its implications for clinical acceptance of PACS. SPIE Medical Imaging 1234: 670-675, 1990

10. Fisher P, Grover B, Brauer G, et al: Digital image display station performance requirements based on physician experience with a prototype system. J Digital Imaging 2:150-155, 1989

11. Roger E, Goldberg M, Dillon RF: Image organization and navigation strategies for a radiological work station. J Digital Imaging 2:229-244, 1989

12. Haynor DR, Saarinen AO: The old study and the correlative study: Implications for PACS. SPIE Medical Imaging III 1093:10-12, 1989

13. Hedgcock MW, Levitt T, Karshat W, et al: Database requirements for PACS. SPIE Medical Imaging IV 1234:588592,1990

14. Sheng OR, Wang H, Garcia HC: IRES-Image retrieval expert system. SPIE Medical Imaging IV 1234:832 841,1990

15. D Beard: Designing a radiology workstation: a focus on navigation during the interpretation task. J Digital Imaging 3:152-163, 1990

16. Saarinen AO, Wilson MC, Iverson SC, et al: PACS economics and the referring physician. SPIE Medical Imaging 1234:806-816, 1990

17. Arenson RL, Seshadri SB, Kundel HL, et al: Clinical evaluation of a medical image management system for chest images. Am J Roentgenol 150:55-59, 1988

18. Beard D, Parrish D, Stevenson D: A cost analysis of film image management and four PACS based on different network protocols. J Digital Imaging 3:108-118, 1990

19. National Electrical Manufacturers Association: Digital imaging and communication. ACR-NEMA Standards Publication, No. 300-1985, 1985, and No. 300-1988, 1988
20. Keller PA: Cathode-ray tube displays for medical imaging. J Digital Imaging 3:15-25, 1990

21. Cox GG, Cook LT, McMillan IH, et al: Chest radiography: comparison of high-resolution digital displays with conventional and digital film. Radiology 176:771-776, 1990

22. Slasky BS, Gur D, Good WF, et al: Receiver operating characteristic analysis of chest image interpretation with conventional, laser-printed, and high-resolution workstation images. Radiology 174:775-780, 1990

23. Murphey MD, Bramble JM, Cook LT, et al: Nondisplaced fractures: spatial resolution requirements for detection with digital skeletal imaging. Radiology 174:865-870, 1990

24. Wegryn SA, Piraino DW, Richmond BJ, et al: Comparison of digital and conventional musculoskeletal radiography: an observer performance study. Radiology 175:225228,1990

25. Murphey MD: Digital skeletal radiography: spatial resolution requirements for detection of subperiosteal resorption. Am J Roengtenol 152:541-546, 1989

26. Murphey MD, Huang HK, Siegel EL, et al: Clinical experience in the use of photostimulable phosphor radiographic systems. Invest Radiol 26:590-597, 1991

27. Gross GW, Ehrlich SM, Wang Y: Diagnostic quality of portable abdominal radiographs in neonates with necrotizing enterocolitis. Am J Roentgenol 154:779-783, 1990

28. Erdekian VV, Trombetta SP: Display systems for medical imaging. SPIE Medical Imaging V 1444:151-158, 1991

29. Roehrig H, Blume H, Ji TL, Browne M: Performance tests and quality control of cathode ray tube displays. J Digital Imaging 3:134-145, 1990

30. Beard D, Cromartie R, Creasy J, et al: Experiment comparing image-locating on film and the FILM-PLANE workstation. SPIE Medical Imaging II 914:933-937, 1988

31. Horii S, Horii H, Mun SK, et al: Environmental designs for reading from imaging workstations: ergonomic and architectural features. J Digital Imaging 2:156-162, 1989

32. Ohhashi A, Yamada S, Haruki K, et al: Automatic adjustment of display window (gray-level) for MR images using a neural network. SPIE Medical Imaging V 1444:6374, 1991

33. Murphey MD, Bramble JM, Cox GC, et al: Experience in the use of an image-processing workstation for a photostimulabel phosphor radiographic system. J Digital Imaging 3:60-65, 1990

34. MacMahon H, Doi K, Chan HP, et al: Computeraided diagnosis in chest radiology. $\mathrm{J}$ Thorac Imaging 5:6776,1990

35. Swett HA, Fisher PR, Cohn AI, et al: Expert systemcontrolled image display. Radiology 172:487-493, 1989

36. Piraino D, Richmond B, Schluchter M, et al: Radiology image interpretation system: modified observer performance study of an image interpretation expert system. $J$ Digital Imaging 4:94-101, 1991

37. Apicella PL, Blaine GJ, Jost RG: A prototype of a high-resolution computerized radiology teaching file. J Digital Imaging 4:43-50, 1991 